\title{
A telehealth intervention to increase patient preparedness for surgery: a randomized trial
}

\author{
Gabriela E. Halder ${ }^{1}$ (D) $\cdot$ Amanda B. White ${ }^{1} \cdot$ Heidi W. Brown ${ }^{2} \cdot$ Lauren Caldwell $^{1} \cdot$ Michelle L. Wright $^{1} \cdot$ Dobie L. Giles $^{2}$ • \\ Christine A. Heisler ${ }^{2} \cdot$ Daksha Bilagi $^{1} \cdot$ Rebecca G. Rogers ${ }^{1,3}$
}

Received: 5 March 2021 / Accepted: 26 April 2021 / Published online: 24 May 2021

(C) The International Urogynecological Association 2021

\begin{abstract}
Introduction and hypothesis Methods to increase surgical preparedness in urogynecology are lacking. Our objective was to evaluate the impact of a preoperative provider-initiated telehealth call on surgical preparedness.

Methods This was a multicenter randomized controlled trial. Women undergoing surgery for pelvic organ prolapse and/or stress urinary incontinence were randomized to either a telehealth call $3( \pm 2)$ days before surgery plus usual preoperative counseling versus usual preoperative counseling alone. Our primary outcome was surgical preparedness, as measured by the Preoperative Prepardeness Questionnaire. The Modified Surgical Pain Scale, Pelvic Floor Distress Inventory-20, Patient Global Impressions of Improvement, Patient Global Impressions of Severity, Satisfaction with Decision Scale, Decision Regret Scale, and ClavienDindo scores were obtained at 4-8 weeks postoperatively and comparisons were made between groups.

Results Mean telehealth call time was $11.1 \pm 4.11 \mathrm{~min}$. Women who received a preoperative telehealth call $(n=63)$ were significantly more prepared for surgery than those who received usual preoperative counseling alone $(n=69) ; 82.5$ vs $59.4 \%$, $p<0.01)$. A preoperative telehealth call was associated with greater understanding of surgical alternatives $(77.8 \mathrm{vs} 59.4 \%, p=$ $0.03)$, complications $(69.8 \mathrm{vs} 47.8 \%, p=0.01)$, hospital-based catheter care ( $54 \mathrm{vs} 34.8 \%, p=0.04)$ and patient perception that nurses and doctors had spent enough time preparing them for their upcoming surgery $(84.1 \mathrm{vs} 60.9 \%, p<0.01)$. At $4-8$ weeks, no differences in postoperative and patient reported outcomes were observed between groups (all $p>0.05$ ).

Conclusions A short preoperative telehealth call improves patient preparedness for urogynecological surgery.
\end{abstract}

Keywords Telemedicine $\cdot$ Surgery $\cdot$ Counseling $\cdot$ Preoperative care

Clinical trial registration Registered with ClinicalTrials.gov

Date of registration: March 26, 2019

Date of initial participant enrollment: June 5, 2019

URL: https://clinicaltrials.gov/ct2/show/record/NCT03890471

Clinical trial identification number: NCT03890471

Presented at the American Urogynecologic Society's Pelvic Floor Disorders Week Annual Meeting, Virtual Meeting, 8-10 October 2020

Gabriela E. Halder

gabrielahalder@gmail.com

1 Department of Women's Health, University of Texas Austin Dell Medical School, Medical Park Tower, 1301 W. 38th St., Suite 705, Austin, TX 78705, USA

2 Department of Obstetrics and Gynecology, Division of Female Pelvic Medicine and Reconstructive Surgery, University of Wisconsin-Maddison, Maddison, WI, USA

3 Department of Obstetrics and Gynecology, Albany Medical Center, Albany, NY, USA

\section{Introduction}

Approximately $25 \%$ of US women are affected by pelvic floor disorders $[1,2]$. The management of these non-lifethreatening conditions is patient centered and focused on patient-desired outcomes. Twenty percent of US women will seek surgical management of their pelvic floor disorders by the age of 80 [3]. The appropriate selection and counseling of patients seeking surgery is important to ensure that desired outcomes can be achieved through a surgical route. Unrealistic surgical expectations result in patient-reported failure in the setting of otherwise quantitatively measured success [4].

Surgical preparedness encompasses appropriate patient understanding of procedural goals, risks, benefits, and expectations of the post-procedural recovery and therapeutic outcome. Surgical preparedness improves patient satisfaction and perioperative outcomes. Prepared women undergoing 
pelvic reconstructive surgery have better Pelvic Floor Distress Inventory-20 (PFDI-20), Patient Global Impressions of Improvement (PGI-I), and Patient Global Impressions of Severity (PGI-S) scores, are less likely to report perioperative complications, and have greater satisfaction than unprepared women at 3 and 12 months postoperatively [4, 5].

Despite improved outcomes among women with increased surgical preparedness, most interventions to increase surgical preparedness have not been successful including video education prior to surgery, peer-centered counseling, or peer support groups [6-8].

Repetition and repeated contact improves patient recall of medical information and is particularly helpful in older patients who have great difficulties in retaining information [9, 10]. A planned preoperative telehealth call prior to surgery offers women an additional opportunity to review surgical and perioperative expectations with their provider and ask questions. We hypothesized that an opportunity for patients to review preoperative counseling with their provider after they have had time to read written material and reflect on their surgery would be beneficial in aiding comprehension and would improve surgical preparedness.

The primary aim of this study was to evaluate the impact of a provider-initiated telehealth call 3 days before surgery on surgical preparedness for women undergoing surgery for stress urinary incontinence (SUI) and/or pelvic organ prolapse (POP). We secondarily sought to determine the impact of a preoperative telehealth call on surgical satisfaction, postoperative pain scores, surgical complications, and patient-reported outcomes $4-8$ weeks after surgery. Our hypothesis was that women who received a preoperative telehealth call would report increased preparedness for surgery and that they would have greater surgical satisfaction, report fewer complications, and have higher scores on postoperative patient-reported outcomes.

\section{Materials and methods}

This was a randomized control trial to evaluate whether an additional provider-initiated telehealth call plus usual preoperative counseling versus usual preoperative counseling alone increased the rate of surgical preparedness among women undergoing surgery for POP and/or SUI. The study was approved by the University of Texas Institutional Review Board (2018-12-0057) and registered with ClinicalTrials.gov (Name of trial registry: Telephone Intervention to Increase Patient Preparedness and Satisfaction Trial [TIPPS]: A Randomized Control Trial; Registration number: NCT03890471) prior to enrollment of participants. Women were recruited from urogynecology surgery clinics associated with two major tertiary referral teaching hospitals. Inclusion criteria were women over the age of 18 years who could read and speak
English and were scheduled to undergo surgery for SUI and/or POP. Concomitant procedures were permitted. Women who were unable to participate in a telehealth call (owing to dementia, a hearing disability, etc.) or scheduled to undergo surgery in less than 3 days were excluded. Enrollment and consent were completed during preoperative in-person visits.

Data gathered at enrollment included baseline characteristics, PGI-S [11], PFDI-20 [12], Modified Surgical Pain Scale (SPS) [12], medical and surgical history, and Pelvic Organ Prolapse Quantification (POP-Q) measurements. Preoperative counseling was completed by a Female Pelvic Medicine and Reconstructive (FPMRS) attending. A preoperative counseling checklist was used to standardize counseling at the in-person preoperative visit (see Fig. 2). Components included surgical purpose, alternatives, benefits, risks/complications, and postoperative expectations. We also included a section on preoperative health optimization based on urogynecology Enhanced Recovery After Surgery (ERAS) Society protocols with recommendations on the management of chronic medical conditions, alcohol and tobacco cessation, and daily exercise $[13,14]$.

Women were randomized to either a provider-initiated telehealth call plus usual preoperative counseling or usual preoperative counseling alone by an online study randomizing program (https://app.studyrandomizer.com) [15]. A varying permutated block size of 4-8 with equal probability of allocation to either arm was used. The allocation sequence was generated by the online program and the randomization arm for each participant was visible to research personnel only at the time of randomization, which took place 7 days $( \pm$ a 3 -day window) before surgery. Women randomized to the telehealth call were contacted 3 days ( \pm a 2 -day window) prior to surgery. During enrollment, participants were advised that they would only receive a telehealth call if they were randomized to that arm of the study. After randomization, women were contacted directly by their provider. Telehealth calls were completed by a single FPMRS attending or fellow surgeon who was part of the patient's care team. All telehealth calls were performed via telephone (not video) call. To standardize calls, a semi-scripted checklist that covered the same items as the preoperative checklist was used.

On the day of surgery, participants completed the Preoperative Patient Questionnaire (PPQ) in the preoperative holding area and were asked whether they had obtained additional educational information from outside sources. Surgical information, including type of surgery, route of anesthesia, use of catheter upon discharge from the hospital, estimated blood loss (EBL), and adverse events, were recorded. On the first postoperative day, the Modified SPS was administered. Women were followed up in clinic 4-8 weeks after surgery and completed the PGI-S, PFDI-20, Modified SPS, Decision Regret Scale (DRS) [16], Satisfaction with Decision Scale (SDS) [16], and the Patient Global Impressions of 
Improvement (PGI-I) scale [11]. Adverse events that occurred up to 8 weeks following surgery were abstracted from clinical charts.

The primary outcome for this study was patient preparedness for surgery to correct POP and/or SUI as measured by the Preoperative Prepardeness Questionnaire (PPQ) question number 11 (Q11), "Overall, I feel prepared for my upcoming surgery." Participants who responded "strongly agree" were considered prepared, whereas all other responses to the fivepoint Likert scale were considered not prepared. The PPQ is an 11-item questionnaire developed by Kenton et al. [4]. Although not validated, this survey has been used in several urogynecological studies assessing surgical preparedness with the same cutoff score for question 11 [6-8]. Currently, there are no validated measures to assess surgical preparedness in urogynecology.

Secondary outcomes included surgical satisfaction as measured by the SDS and DRS, postoperative pain scores as measured by the Modified SPS, surgical complications and patient-reported outcomes as measured by the PFDI-20, PGI-S, and PGI-I. For the PGI-I, the original seven responses were re-categorized into three groups owing to low numbers across the responses: better (very much better, much better, a little better), no change, and worse (very much worse, much worse, a little worse). Objective postoperative change in pelvic floor dysfunction from baseline was ascertained using the change in PGI-S and PFDI-20 from baseline. Change in PGI$\mathrm{S}$ was calculated by assigning a value of 1 to normal, 2 to mild, 3 to moderate, and 4 to severe.

To estimate sample size, we assumed that $58 \%$ of participants in the non-intervention group (preoperative counseling alone) would feel prepared for surgery, based on prior studies evaluating surgical preparedness in urogynecology [4]. We also assumed that $80 \%$ of participants in the intervention group would feel prepared for surgery based on the results from the only successful intervention in urogynecology to increase surgical preparedness [17]. In this non-randomized trial of 36 women, a group shared appointment prior to placement of sacral neuromodulation for overactive bladder was significantly associated with greater surgical preparedness than standard office counseling $(78.9 \%$ vs $29.4 \%, p=0.0028)$. Using an alpha of 0.05 and a beta of 0.20 , a total of 136 participants were needed to detect a difference of $22 \%$ between intervention arms. Assuming a $10 \%$ attrition rate, we sought to enroll a total of 150 participants (75 in each arm). We planned an intentto-treat analysis.

All data were entered into RedCap and analyzed using $\mathrm{R}$ version 3.6.1. Continuous variables were analyzed as means and standard deviations or medians and interquartile ranges based on distributions. Normality was assessed with both histograms and tests for normality. Variables were compared using Welch's $t$ tests or Wilcoxon rank-sum tests also based on distributions. Frequencies and percentages were calculated for categorical variables and compared using the Chi-squared or Fisher's exact test as appropriate. A $p$ value less than 0.05 was considered statistically significant.

\section{Results}

Between June 2019 and May 2020, a total of 150 patients were enrolled and 143 women were randomized; 73 were allocated to usual preoperative counseling alone and 70 were allocated to usual preoperative counseling plus a telehealth call. Seventeen surgeries were canceled, 7 before randomization and 10 after. At the completion of the study, it was determined that one patient did not meet the inclusion criteria and had been enrolled inappropriately. In the intervention arm, three participants did not receive a telehealth call; their data were included in the intentionto-treat analysis. A total of 132 women were included in our final analysis (telehealth call: $n=63$, usual preoperative counseling alone: $n=69$ ). Figure 1 shows the flow of participants through the study.

For all women, the mean age was $57.9 \pm 13.3$ years, $92 \%$ were white, $27 \%$ were Hispanic, $64 \%$ had private insurance, $61 \%$ were married, and $61 \%$ had longer than a high school education. The diagnosis of POP alone, urinary incontinence alone (UI), and POP with UI were 20\%, $24 \%$, and $56 \%$ respectively. Eighteen percent of patients had undergone prior pelvic floor reconstructive surgery. Groups did not vary in baseline characteristics (Table 1). The mean time spent on a telehealth call was $11.1 \pm$ $4.11 \mathrm{~min}$.

A significantly higher proportion of women who received an additional preoperative telehealth call plus usual preoperative counseling were prepared for surgery than women in the group that received usual preoperative counseling alone ( 83 vs $59 \%, p<0.01$; Table 2 ). A preoperative telehealth call also resulted in greater understanding of the alternatives ( 78 vs $59 \%, p=0.03$ ) and complications to the planned surgery ( 70 vs $48 \%, p=0.01$ ). More participants who received a telehealth call felt prepared to cope with a catheter after the surgery whereas in the hospital (54 vs $35 \%, p=0.04$ ) and that their nurses and doctors and spent enough time preparing them for their upcoming surgery ( 84 vs $61 \%, p<0.01)$. Participants in both groups felt least prepared for potential home-based catheter care (33 vs $21 \%, p=0.1$ ).

An equal number of women in each group obtained information about their surgery from a source other than their doctor (36 vs 38\%,p=1). Other sources of information included the internet $(25 \%)$, television (2\%), pamphlets $(9 \%)$, friends $(13 \%)$, family (7\%), and other healthcare professionals $(5 \%)$. 
Fig. 1 Enrollment, randomization, and analysis of research participants
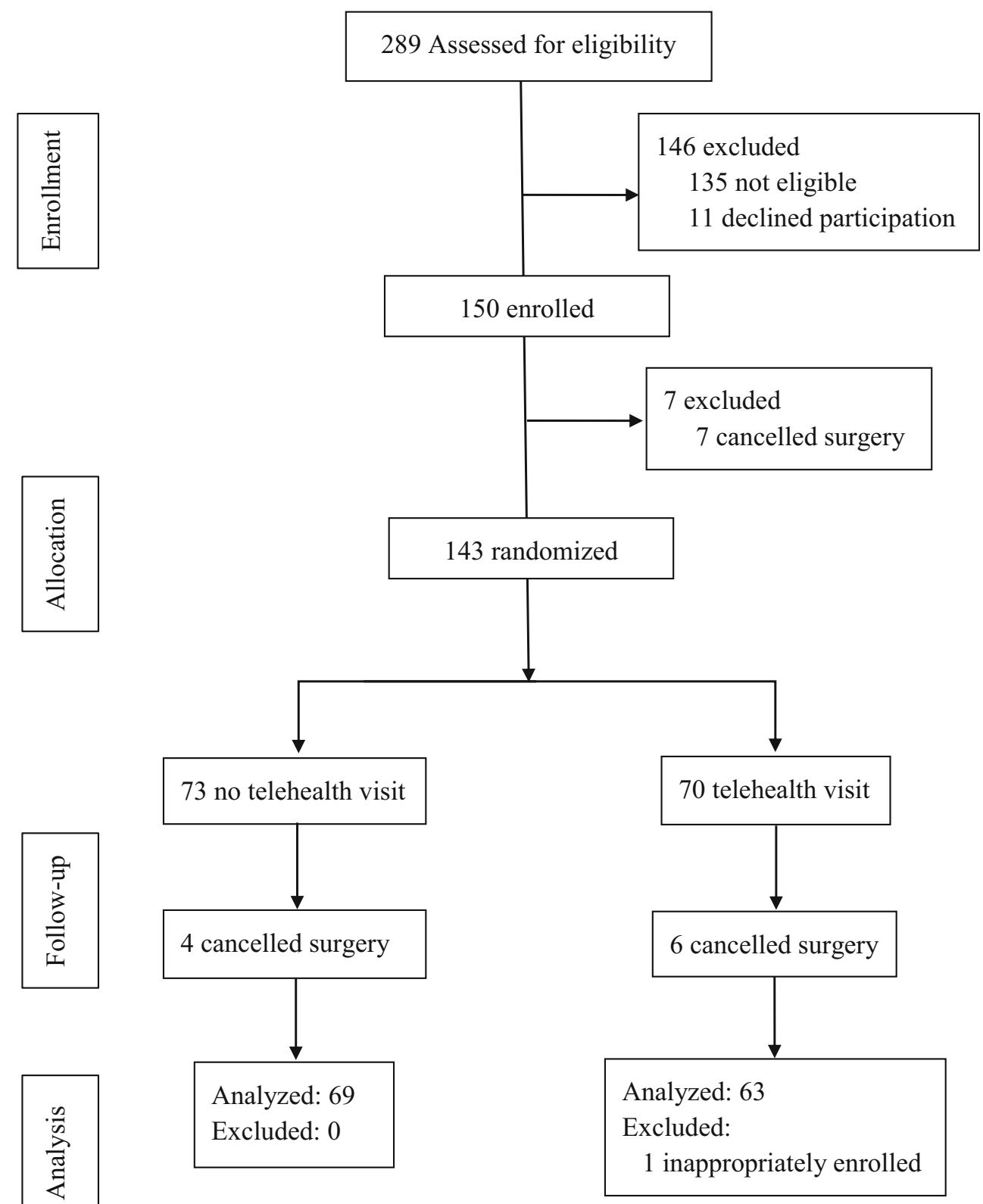

All participants underwent surgery under general anesthesia. There were no differences in the surgical procedures performed, EBL, and Clavien-Dindo scores for immediate surgical complications between the groups (Table 3). Thirty-six percent of participants were discharged home with a catheter, with no differences between the groups (43 vs $30 \%, p=$ 0.149).

Postoperatively, there were no differences in the scores for the modified SPS (postoperative day 1 and 4-8 weeks postoperative scores), PFDI-20, PGI-I, or PGI-S, SDS, DRS, or Clavien-Dindo between the groups (all $p>0.05$; Table 4 ). There were no differences in the changes between pre- and post-operative PFDI-20 and PGI-S scores between the groups (all $p>0.05$ ).

\section{Discussion}

An additional preoperative telehealth call improved patient preparedness for urogynecological surgery. No differences were observed between groups in postoperative patient reported outcomes; this finding may be explained by insufficient power and/or short-term follow-up. To our knowledge, our study is one of the first randomized interventional trials to increase surgical preparedness in patients undergoing urogynecological surgery.

A handful of studies in urogynecology have evaluated the efficacy of various interventions at increasing surgical preparedness with limited results. Similar to our study, preparedness was measured in these studies using a single question on 
Table 1 Baseline characteristics

\begin{tabular}{|c|c|c|c|}
\hline & $\begin{array}{l}\text { Telehealth Call } \\
(n=63)\end{array}$ & $\begin{array}{l}\text { No telehealth Call } \\
(n=69)\end{array}$ & $p$ value \\
\hline Age (years) & $57.6 \pm 13.1$ & $58.2 \pm 13.6$ & 0.78 \\
\hline BMI $\left(\mathrm{kg} / \mathrm{m}^{2}\right)$ & $29.3 \pm 7.1$ & $28.4 \pm 6.7$ & 0.44 \\
\hline Race & & & 0.17 \\
\hline White & $61(97)$ & $61(88)$ & \\
\hline Other & $2(3)$ & $6(9)$ & \\
\hline Missing & $0(0)$ & $2(3)$ & \\
\hline Ethnicity & & & 0.95 \\
\hline Hispanic & $17(27)$ & $18(27)$ & \\
\hline Missing & $2(3)$ & $2(3)$ & \\
\hline Health insurance & & & 0.26 \\
\hline Private & $39(62)$ & $45(65)$ & \\
\hline Government issued & $24(38)$ & $21(30)$ & \\
\hline Self-pay & $0(0)$ & $3(5)$ & \\
\hline \multicolumn{4}{|l|}{ Married/partner status } \\
\hline Single & $22(35)$ & $28(41)$ & 0.53 \\
\hline Married & $41(65)$ & $40(58)$ & \\
\hline Missing & $0(0)$ & $1(2)$ & \\
\hline \multicolumn{4}{|l|}{ Highest level of education } \\
\hline High school or less than & $29(46)$ & $22(32)$ & 0.13 \\
\hline Greater than high school & $34(54)$ & $46(67)$ & \\
\hline Missing & $0(0)$ & $1(2)$ & \\
\hline Charlson comorbidity Index & $1.9 \pm 1.8$ & $2.3 \pm 2.2$ & 0.27 \\
\hline Preoperative modified SPS & $1.6 \pm 2.4$ & $1.9 \pm 2.4$ & 0.30 \\
\hline Diagnosis & & & 0.15 \\
\hline POP alone & $12(19)$ & $14(20)$ & \\
\hline UI alone & $20(32)$ & $12(17)$ & \\
\hline POP and UI & $31(49)$ & $43(62)$ & \\
\hline POP stage & & & 0.84 \\
\hline Stage 2 & $19(44)$ & $28(51)$ & \\
\hline Stage 3 & $22(51)$ & $24(44)$ & \\
\hline Stage 4 & $2(5)$ & $3(6)$ & \\
\hline \multicolumn{4}{|l|}{ PGI-S } \\
\hline Normal & $2(3)$ & $3(4)$ & 0.31 \\
\hline Mild & $6(10)$ & $10(15)$ & \\
\hline Moderate & $31(49)$ & $29(41)$ & \\
\hline Severe & $24(38)$ & $24(35)$ & \\
\hline Preoperative PFDI-20 & $103.6 \pm 57.8$ & $103.7 \pm 55.3$ & 0.92 \\
\hline POPDI-6 & $36.1 \pm 23.8$ & $36.9 \pm 22.7$ & 0.85 \\
\hline UDI-6 & $46.2 \pm 27.1$ & $40.6 \pm 26.0$ & 0.24 \\
\hline CRADI-8 & $20.2 \pm 21.2$ & $25.2 \pm 22.4$ & 0.17 \\
\hline Prior pelvic reconstructive surgery for POP and/or SUI & $7(11)$ & $17(25)$ & 0.06 \\
\hline
\end{tabular}

Data are represented as mean $\pm \mathrm{SD}$ or $n(\%)$ unless otherwise specified

$B M I$ body mass index, SPS Surgical Pain Scale, $P O P$ pelvic organ prolapse, UI urinary incontinence, $P G I-S$ Patient Global Impression of Severity, PFDI-20 Preoperative Pelvic Floor Distress Inventory-20, POPDI-6 Pelvic Organ prolapse Distress Inventory 6, UDI-6 Urinary distress Inventory 6, CRADI-8 Colorectal-Anal distress Inventory 8

the PPQ. A randomized trial comparing a preoperative educational video as an adjunct to usual care $(n=52)$ versus usual care alone $(n=48)$ for patients undergoing a sacrocolpopexy found similar rates of preparedness in the two groups (video: 
Table 2 Percentage of patients reporting preparedness on each item of the PPQ by treatment arm

\begin{tabular}{|c|c|c|c|}
\hline PPQ question & $\begin{array}{l}\text { Telehealth Calll } \\
(N=63)\end{array}$ & $\begin{array}{l}\text { No Telehealth Call } \\
(N=69)\end{array}$ & $\begin{array}{l}p \\
\text { value }\end{array}$ \\
\hline 1. I know about the alternatives to the planned surgery & $49(78)$ & $41(59)$ & $0.03 *$ \\
\hline 2. I understand the purpose of the planned surgery (what this surgery can accomplish) & $51(81)$ & $50(73)$ & 0.31 \\
\hline 3. I understand the benefits of the planned surgery (how this surgery should help me) & $52(83)$ & $50(73)$ & 0.21 \\
\hline $\begin{array}{l}\text { 4. I understand the risks of the planned surgery (what the chances are of something not going the way } \\
\text { my doctor and I want it to go) }\end{array}$ & $46(73)$ & $39(57)$ & 0.07 \\
\hline 5. I understand the complications of the planned surgery (what problems can come from this surgery) & $44(70)$ & $33(48)$ & $0.01 *$ \\
\hline 6. I feel prepared about what to expect after surgery while I am in the hospital & $42(67)$ & $34(49)$ & 0.05 \\
\hline 7. I feel prepared about what to expect after surgery when I am at home & $36(57)$ & $27(39)$ & 0.06 \\
\hline 8. I feel prepared to cope with a catheter after the surgery while I am in the hospital & $34(54)$ & $24(35)$ & $0.04 *$ \\
\hline 9. I feel prepared to cope with a catheter after the surgery while I am at home & $21(33)$ & $14(21)$ & 0.12 \\
\hline 10. My doctors and nurses have spent enough time preparing me for my upcoming surgery & $53(84)$ & $42(61)$ & $<0.01 *$ \\
\hline 11. Overall I feel prepared for my upcoming surgery & $52(83)$ & $41(59)$ & $<0.01 *$ \\
\hline
\end{tabular}

Women were categorized as prepared if they responded "strongly agree" on a five-point Likert scale

*Denotes statistical significance

Data are represented as $\mathrm{n}(\%)$

71.1 vs usual care: $68.8 \%, p=0.79)$ [6]. A randomized trial comparing a peer-centered $(n=32)$ versus a standard physician-centered $(n=30)$ preoperative counseling video for patients undergoing midurethral sling placement also found no differences in preparedness between groups (peercentered group: 75 vs physician-centered group: $73 \%, p=$ 1.00) [7]. A prospective cohort comparing a peer support group ( $n=113$ ) versus usual one-to-one care $(n=55)$ for women undergoing surgery for POP and/or SUI found similar rates of preparedness in the two groups (peer support: $66 \mathrm{vs}$ usual care: $63 \%, p=0.9$ ) [8]. The only other intervention shown to successfully increase surgical preparedness is group shared appointments. Based on a non-randomized case series for patients undergoing sacral neuromodulation, patients attending a group shared appointment $(n=19)$ were more prepared than those attending standard office counseling $(n=17)$ (shared: 42 vs standard: 12\%, $p=0.043$ ) [17]. Our study contributes to the growing body of knowledge on successful preoperative interventions that increase surgical preparedness in urogynecology.

Table 3 Surgical characteristics and outcomes

\begin{tabular}{|c|c|c|c|}
\hline & $\begin{array}{l}\text { Telehealth Call } \\
(N=63)\end{array}$ & $\begin{array}{l}\text { No Telehealth Call } \\
(N=69)\end{array}$ & $p$ value \\
\hline $\mathrm{EBL}^{\mathrm{a}}$ & $67.5(50,100)$ & $50.0(50,100)$ & 0.82 \\
\hline Hysterectomy & $27(43)$ & $24(35)$ & 0.38 \\
\hline Prolapse surgery ${ }^{\mathrm{b}}$ & & & 0.55 \\
\hline Sacrocolpopexy & $19(30)$ & $18(26)$ & \\
\hline Uterosacral ligament suspension & $14(22)$ & $17(25)$ & \\
\hline Colpocleisis & $3(9)$ & $6(5)$ & \\
\hline Sling $^{c}$ & $46(73)$ & $41(59)$ & 0.14 \\
\hline Clavien-Dindo score & & & 1.00 \\
\hline Grade I & $23(37)$ & $24(35)$ & \\
\hline Grade III & $3(5)$ & $3(4)$ & \\
\hline Discharged with a catheter & $27(43)$ & $21(30)$ & 0.15 \\
\hline
\end{tabular}

$E B L$ estimated blood loss

Data are represented as $n(\%)$ unless otherwise specified

${ }^{\text {a }}$ Data represented as median (interquartile range)

${ }^{\mathrm{b}}$ Patients had a combination of prolapse only, prolapse and sling, and sling only procedures and therefore the total number of procedures do not equal $100 \%$ 
Table 4 Postoperative outcomes

\begin{tabular}{|c|c|c|c|}
\hline & $\begin{array}{l}\text { Telehealth } \\
\text { Call } \\
(N=63)\end{array}$ & $\begin{array}{l}\text { No Telehealth Call } \\
(N=69)\end{array}$ & $p$ value \\
\hline \multicolumn{4}{|c|}{ 1-Day postoperative outcomes } \\
\hline Modified SPS & $4.7 \pm 2.4$ & $4.2 \pm 2.5$ & 0.27 \\
\hline \multicolumn{4}{|c|}{ 4-8 Weeks postoperative outcomes } \\
\hline Modified SPS & $1.2 \pm 2.2$ & $0.9 \pm 1.5$ & 0.84 \\
\hline PFDI- $20^{\mathrm{a}}$ & $25(6.8,73.4)$ & $20.8(0,58.3)$ & 0.37 \\
\hline POPDI- $6^{\mathrm{a}}$ & $4.2(0,16.7)$ & $8.3(0,16.7)$ & 0.75 \\
\hline UDI- $6^{\mathrm{a}}$ & $8.3(0,29.2)$ & $6.3(0,16.7)$ & 0.27 \\
\hline CRADI- $8^{\mathrm{a}}$ & $6.3(0,18.8)$ & $6.3(0,18.8)$ & 0.63 \\
\hline PGI-I & & & 1.00 \\
\hline Better & $60(95)$ & $61(88)$ & \\
\hline No change & $0(0)$ & $0(0)$ & \\
\hline Worse & $2(3)$ & $2(3)$ & \\
\hline PGI-S & & & 0.26 \\
\hline Normal & $46(73)$ & $47(68)$ & \\
\hline Mild & $8(13)$ & $13(19)$ & \\
\hline Moderate & $6(10)$ & $3(4)$ & \\
\hline Severe & $2(3)$ & $0(0)$ & \\
\hline SDS & $4.8 \pm 0.5$ & $4.8 \pm 0.4$ & 0.39 \\
\hline DRS & $1.3 \pm 0.6$ & $1.2 \pm 0.6$ & 0.41 \\
\hline $\begin{array}{l}\text { Clavien-Dindo } \\
\text { category }\end{array}$ & & & 0.91 \\
\hline Grade I & $8(13)$ & $5(7)$ & \\
\hline Grade II & $17(27)$ & $20(29)$ & \\
\hline Grade III & $4(6)$ & $4(6)$ & \\
\hline \multicolumn{4}{|c|}{ Postoperative changes from baseline } \\
\hline Change in PFDI-20 & $62.7 \pm 52.2$ & $69.7 \pm 57.6$ & 0.49 \\
\hline Change in POPDI-6 & $23.8 \pm 23.9$ & $26.3 \pm 23.6$ & 0.58 \\
\hline Change in UDI-6 & $28.7 \pm 31.0$ & $27.7 \pm 25.8$ & 0.84 \\
\hline Change in CRADI-8 & $20.2 \pm 21.2$ & $25.2 \pm 22.3$ & 0.17 \\
\hline Change in PGI-S & $1.82 \pm 1.1$ & $1.82 \pm 1.1$ & 0.90 \\
\hline
\end{tabular}

SPS Surgical Pain Scale, PFDI-20 Preoperative Pelvic Floor Distress Inventory-20, POPDI-6 Pelvic Organ prolapse Distress Inventory 6, UDI-6 Urinary distress Inventory 6, CRADI-8 Colorectal-Anal distress Inventory 8, PGI-I Patient Global Impression of Improvement, PGI-S Patient Global Impression of Severity, DRS Decision Regret Scale, SDS Satisfaction with Decision Scale

Data are represented as mean $\pm \mathrm{SD}$ or $n(\%)$ unless otherwise specified

${ }^{\mathrm{a}}$ Data represented as median (interquartile range)

Our study also supports the ERAS Society's published guidelines for pre- and intra-operative care in gynecological/ oncological surgery [14]. The preoperative counseling checklist used in our study incorporated ERAS Society recommendations such as the adequate management of chronic medical conditions, cessation of alcohol and tobacco consumption, and 30 min of walking daily. In their section on "Preadmission information, education and counseling," preoperative counseling has a strong recommendation grade for use despite a low-quality evidence rating. The ERAS Society recommends preoperative counseling despite a lowquality evidence rating because "most studies show that counseling provides beneficial effects with no evidence of harm." The findings of our study support ERAS Society recommendations for counseling, as all participants received at least routine preoperative counseling and both arms of the study demonstrated surgical preparedness.

Kenton et al. [4] found that at 3 months postoperatively, prepared women were more likely have improved PGI-I scores ( 68 vs $32 \%, p=0.003)$, report greater satisfaction with their surgery ( $77 \mathrm{vs} 23 \%, p<0.05$ ), and have improved postoperative POPDI (0 [0-35], vs $8[0-46], p=0.02)$ and UDI $(0$ $[0-33]$ vs $13[0-67], p=0.02$ ) scores, whereas objective measures of cure did not differ by preparedness. The process of evaluating surgical preparedness does allow for negotiation of patient expectations to ensure alignment between surgeon and patient, which may help to explain higher scores on patientreported outcomes.

The success of a preoperative telehealth call on surgical preparedness may hinge on the additional touch point with a provider. Of the medical information provided by healthcare practitioners, $40-80 \%$ is forgotten immediately by patients. Furthermore, the greater the amount of information presented, the lower the proportion correctly recalled [18, 19]. Information acquisition in a stressful clinical setting hampers memory formation [20]. Evidence exists that repetition of medical information improves memory in both older and younger populations [10]. Prior interventions aimed at increasing surgical preparedness in urogynecology relied on an intervention at the time of initial counseling for surgery [6-8]. To our knowledge, our intervention is the first to provide an additional opportunity for patients to repeat preoperative counseling with their provider after they have had time to review written material and reflect on their surgery; an additional touch point may be beneficial in aiding comprehension.

Our study is limited by its short length of follow-up of 4-8 weeks after surgery. This short time frame may have limited our ability to detect significant differences in postoperative patient-reported outcomes; additionally, our study was not sufficiently powered to detect such differences. Strengths of our study include its randomized design, the use of standardized checklists to ensure that counseling was delivered consistently across sites and providers, and our use of the PPQ. Although not fully validated, the PPQ has been used extensively to evaluate surgical preparedness in urogynecology, making it feasible for our findings to be compared with the body of existing literature around improving surgical preparedness. Currently, there are no validated questionnaires assessing surgical preparedness in women undergoing urogynecological surgery. 
Fig. 2 Preoperative counseling checklist used to standardize counseling between providers

\begin{tabular}{|c|l|c|}
\hline $\begin{array}{c}\text { Item } \\
\text { number }\end{array}$ & \multicolumn{1}{c|}{ Category } & $\underline{\underline{\text { Check if }}}$ \\
\hline 1 & Pomplete \\
\hline 2 & Alternatives to the planned surgery & $\square$ \\
\hline 3 & Surgical benefits & $\square$ \\
\hline 4 & Surgical risks (examples: infection, bleeding, injury to surrounding organs) & $\square$ \\
\hline 5 & Potential surgical complications (examples: mesh erosion, voiding dysfunction) & $\square$ \\
\hline 6 & Presurgical health optimization & $\square$ \\
\hline & Management of chronic medical conditions (if applicable) & $\square$ \\
\hline & Advise cessation of alcohol and tobacco 4-6 weeks before surgery (if applicable) & $\square$ \\
\hline & 30 minutes of walking daily & $\square$ \\
\hline 7 & Postoperative expectations (examples: Foley, pain, activity restrictions, diet) & $\square$ \\
\hline
\end{tabular}

Intervention blinding was not feasible from the patient's standpoint owing to the nature of the intervention being a telehealth call. We did not see the need to blind providers as randomization was performed after usual preoperative counseling. Finally, although we conceived this study before the COVID-19 global pandemic changed healthcare delivery in the US dramatically, its findings are perhaps even more applicable in our current context, where we are expanding the use of telehealth in our practices.

As mentioned previously, prepared patients have better postoperative outcomes. Our study was not powered to detect differences between groups in postoperative outcomes. Larger enrollment numbers and longer follow-up time may allow us to detect such differences and fully assess the impact of a telehealth call on the urogynecological perioperative experience.

In our study, the telehealth calls were performed by an FPMRS surgeon (either an attending or a fellow) who was part of the patient's care team. We felt that an additional review of appropriate patient expectations by an individual who had detailed experience with the surgery was at the core of the intervention's success. Future research is needed to determine if a preoperative telehealth call performed by support staff (including medical assistants, nurses, and advanced practice providers) is equally efficacious in increasing preparedness.

In conclusion, a brief telehealth call in addition to standard preoperative counseling improved patient preparedness for urogynecological surgery. Although longer term and larger prospective studies will determine the impact of this intervention on patient-reported outcomes postoperatively, we suggest the use of a preoperative telehealth call (guided by the checklist in Fig. 2) for urogynecology surgeons seeking to increase their patient's surgical preparedness.

Supplementary Information The online version contains supplementary material available at https://doi.org/10.1007/s00192-021-04831-w

Funding Funding for this study was obtained from the Scientific Education and Research Fund at the University of Texas Austin Dell Medical School.

\section{Declarations}

Conflicts of interest None.

\section{References}

1. Wu JM, Vaughan CP, Goode PS, et al. Prevalence and trends of symptomatic pelvic floor disorders in U.S. women. Obstet Gynecol. 2014;123(1):141-8.

2. Sung VW, Hampton BS. Epidemiology of pelvic floor dysfunction. Obstet Gynecol Clin N Am. 2009;36(3):421-43.

3. Wu JM, Matthews CA, Conover MM, et al. Lifetime risk of stress urinary incontinence or pelvic organ prolapse surgery. Obstet Gynecol. 2014;123(6):1201-6.

4. Kenton K, Pham T, Mueller E, et al. Patient preparedness: an important predictor of surgical outcome. Am J Obstet Gynecol. 2007;197(6):654.e651-6.

5. Brubaker L, Litman HJ, Rickey L, et al. Surgical preparation: are patients "ready" for stress urinary incontinence surgery? Int Urogynecol J. 2014;25(1):41-6.

6. Greene KA, Wyman AM, Scott LA, et al. Evaluation of patient preparedness for surgery: a randomized controlled trial. Am J Obstet Gynecol. 2017;217(2):179.e171-7.

7. Krantz TE, Rogers RG, Petersen TR, et al. Peer-centered versus standard physician-centered video counseling for Midurethral sling surgery: a Randomized controlled trial. Female Pelvic Med Reconstr Surg. 2020;26(8):470-6.

8. Madsen AM, Rogers RG, Dunivan GC, et al. Perioperative peer support and surgical preparedness in women undergoing reconstructive pelvic surgery. Int Urogynecol J. 2020;31(6):1123-32.

9. Hall DE, Prochazka AV, Fink AS. Informed consent for clinical treatment. CMAJ. 2012;184(5):533-40.

10. Morrow DG, Leirer VO, Carver LM et al. Effects of aging, message repetition, and note-taking on memory for health information. $\mathrm{J}$ Gerontol B Psychol Sci Soc Sci. 1999;54(6):P369-379.

11. Yalcin I, Bump RC. Validation of two global impression questionnaires for incontinence. Am J Obstet Gynecol. 2003;189(1): 98-101.

12. Barber MD, Janz N, Kenton K, et al. Validation of the surgical pain scales in women undergoing pelvic reconstructive surgery. Female Pelvic Med Reconstr Surg. 2012;18(4):198-204.

13. Carter-Brooks CM, Du AL, Ruppert KM, et al. Implementation of a urogynecology-specific enhanced recovery after surgery (ERAS) pathway. Am J Obstet Gynecol. 2018;219(5):495.e491-10.

14. Nelson G, Altman A, Nick A et al. Guidelines for pre- and intraoperative care in gynecologic/oncology surgery: Enhanced Recovery After Surgery (ERAS@) society recommendations. Gynecol Oncol 2016;140(2):313-22.

15. Study Randomized. Phase Locked Software; 2019.

16. Sung VW, Kauffman N, Raker CA, et al. Validation of decisionmaking outcomes for female pelvic floor disorders. Am J Obstet Gynecol. 2008;198(5):575.e1-6.

17. Firoozi F, Gill B, Ingber MS, et al. Increasing patient preparedness for sacral neuromodulation improves patient reported outcomes 
despite leaving objective measures of success unchanged. J Urol. 2013;190(2):594-7.

18. Anderson JL, Dodman S, Kopelman M, et al. Patient information recall in a rheumatology clinic. Rheumatol Rehabil. 1979;18(1):18-22.

19. McGuire LC. Remembering what the doctor said: organization and adults' memory for medical information. Exp Aging Res. 1996;22(4):403-28.
20. Ley P. Memory for medical information. Br J Soc Clin Psychol. 1979;18(2):245-55.

Publisher's note Springer Nature remains neutral with regard to jurisdictional claims in published maps and institutional affiliations. 